\title{
Postnatal depression and its association with adverse infant health outcomes in low- and middle-income countries: a systematic review and meta-analysis
}

\author{
Abel Fekadu Dadi ${ }^{1,2^{*}}$ (D, Emma R. Miller ${ }^{2}$ and Lillian Mwanri ${ }^{2}$
}

\begin{abstract}
Background: Postnatal Depression (PND) is a mood disorder that steals motherhood and affects the health and development of a newborn. While the impact of PND on motherhood and newborn in developed countries are well described, its epidemiology and health consequences in infant is not well known in middle-and low-income countries. The objective of this review was to determine the burden and association of PND with adverse infant health outcomes in low-and middle- income countries.

Methods: We searched observational studies written in the English language and conducted in middle-and lowincome countries between December 1st, 2007, and December 31st, 2017. The CINHAL, MEDLINE, Emcare, PubMed, Psych Info, and Scopus databases were searched for the following search terms: PND, acute respiratory infection, pneumonia, diarrhea, exclusive breastfeeding, common infant illnesses, and malnutrition. We excluded studies in which the primary outcomes were not measured following a standardized approach. We have meta-analyzed the estimates from primary studies by adjusting for possible publication bias and heterogeneity. The analysis was conducted in Stata 14. The study was registered in PROSPERO protocol number CRD42017082624.

Result: Fifty-eight studies on PND prevalence (among 63,293 women) and 17 studies (among 32,454 infants) on infant health outcomes were included. PND prevalence was higher in the low-income countries (Pooled prevalence $(P P)=25.8 \%$; 95\% Cl: 17.9-33.8\%) than in the middle-income countries (PP $=20.8 \%$; 95\%Cl: 18.4-23.1\%) and reached its peak in five to ten weeks after birth. Poor obstetric history and social support, low economic and educational status, and history of exposure to violence were associated with an increased risk of PND. The risk of having adverse infant health outcomes was 31\% higher among depressed compared to non-depressed postnatal mothers (Pooled relative risk (PRR) $=1.31 ; 95 \% \mathrm{Cl}$ : 1.17-1.48). Malnutrition (1.39; 1.21-1.61), non-exclusive breastfeeding (1.55; 1.39-1.74), and common infant illnesses $(2.55 ; 1.41-4.61)$ were the main adverse health outcomes identified.

(Continued on next page)
\end{abstract}

\footnotetext{
* Correspondence: Fekten@yahoo.com

${ }^{1}$ Institute of Public Health, College of Medicine and Health Sciences,

University of Gondar, Gondar, Ethiopia

${ }^{2}$ College of Medicine and Public Health, Flinders University, Health Sciences

Building, Sturt Road, Bedford Park, Adelaide, SA 5001, Australia
}

(c) The Author(s). 2020 Open Access This article is licensed under a Creative Commons Attribution 4.0 International License, which permits use, sharing, adaptation, distribution and reproduction in any medium or format, as long as you give appropriate credit to the original author(s) and the source, provide a link to the Creative Commons licence, and indicate if changes were made. The images or other third party material in this article are included in the article's Creative Commons licence, unless indicated otherwise in a credit line to the material. If material is not included in the article's Creative Commons licence and your intended use is not permitted by statutory regulation or exceeds the permitted use, you will need to obtain permission directly from the copyright holder. To view a copy of this licence, visit http://creativecommons.org/licenses/by/4.0/. The Creative Commons Public Domain Dedication waiver (http://creativecommons.org/publicdomain/zero/1.0/) applies to the data made available in this article, unless otherwise stated in a credit line to the data. 
(Continued from previous page)

Conclusions: One in four and one in five postnatal mothers were depressed in low and middle-income countries, respectively. Causes of depression could be explained by social, maternal, and psychological constructs. High risk of adverse infant health outcomes was associated with PND. Timely screening of PND and evidence-based interventions were a pressing need in low and middle-income countries.

Keywords: Postnatal depression, Adverse infant health outcomes, Systematic review, Meta-analysis, Low, And middle-income countries

\section{Background}

Worldwide, depression is the most common mental illness and the leading cause of maternal morbidity and disability in the perinatal period $[1,2]$. Postnatal depression (PND) is a crippling mood disorder that steals motherhood [3]. It is characterized by signs and symptoms such as low mood, tiredness, insomnia, irritability, and reduced functioning [4]. The symptoms of PND commences four to six weeks after childbirth [4] and get peak two to three months after birth [3, $5,6]$. The causes of PND are explained by genetics and socio-environmental constructs [7].

Postnatal depression is used to be an issue of Western countries [8], and research focus on this problem has been limited until the relationship found between socioeconomic status and PND [9, 10]. Recent evidence has shown that the prevalence of PND is higher in low-and middle-income countries (range from 7 to 33\%) than high-income countries (range between 13 and 19\%) [5, $11,12]$. Postnatal depression found to be heterogeneous in Africa with lower prevalence in Uganda (7.1\%) and the highest in Zimbabwe (33\%) [13]. Far less is known about PND, although more than $90 \%$ of the world's children are living in low-and middle-income countries [13]. This suggests more concise and updated estimate about PND epidemiology and consequences are pivotal.

Postnatal depression is the second cause of disability next to HIV/AIDS [2], and the most known complication of childbirth [14]. Postnatal depression can increase the cost of the health care system [15], reduce mother's quality of life and workforce in the economy [16]. Postnatally depressed women are at risk of back pain, insomnia, thought of self-harm, suicidal ideation, and poor parenting behavior $[17,18]$. Postnatal depression affects initiation of breastfeeding and effective utilization of available health services $[17,19]$. This could lead to malnutrition and weakened immune systems with further predisposition to illnesses including diarrhea, pneumonia, measles, and other childhood illnesses [12, 20-22].

The identification of women potentially at risk of PND is vital to prevent the onset and subsequent consequences. The previous reviews on PND were descriptive, incomprehensive and inconclusive [11, 23-28], skewed to developed countries [4, 17, 23, 24], and lacked detail quantification of risk factors. The previous reviews about PND effects on adverse infant health outcomes were scarce, descriptive, outdated, and mostly included studies from developed countries [12, 13, 17]. We did this comprehensive review to explore the prevalence and thematically quantify and present most notable risk factors of PND, and to investigate its association with risk of adverse infant health outcomes in low- and middle-income countries.

\section{Methods}

\section{Search strategy}

We searched the CINHAL, MEDLINE, EMCare, PubMed, Psych Info, and Scopus databases for postnatal depression and its effect on adverse infant health outcomes. The following search terms were used: Postnat*, postpart*, depress*, exclusive breastfeeding, pneumonia, common infant illnesses, diarrhea, measles, diarrhea, fever, malnutrition, and infant feeding practices.

\section{Eligibility criteria}

We included observational studies conducted in low and middle-income countries, written in the English language, and published between January 1st, 2007, and December 31st, 2017 with an aim of including only updated data on the topic. Furthermore, studies were included if they fulfilled the following main outcome definitions:- (1) malnutrition was measured using standard indices like wasting, stunting, short stature, or underweight/overweight; (2) age-related infant feeding practice that reported exclusive breastfeeding or complementary feeding; (3) common infant illnesses such as ARI (pneumonia, fever, and cough), malaria, measles, and diarrhea were assessed following the WHO Integrated Management of Newborn and Childhood Illnesses (IMNCI) guideline; (4) measurement of depression was done using a standard and validated screening tools. According to the World Bank Atlas, low-income and middle-income countries are those with the Gross National Income (GNI) per capita of $\leq \$ 1025$ and between $\$ 1026$ to 12,375 . 


\section{Exclusion criteria}

Studies that pooled antenatal and PND scores, had fair to poor quality score on the New Castle Ottawa Scale, studies restricted to very high or low-risk populations, conference proceedings, commentaries, abstracts, reports, and unpublished data were excluded.

\section{Data extraction and study quality assessment}

This section of methodology has been published previously in recent paper [29].

\section{Data analysis}

Estimates from primary studies were reported in prevalence, odds ratios, or relative risks. For the first objective, estimating the overall prevalence of PND, the prevalence extracted from all primary studies were meta-analyzed. For the second objective, identifying prominent risk factors of PND, odds ratios obtained for each risk factor identified from each primary study were meta-analyzed to get a pooled odds ratio for that specific risk factor. For the third objective, investigating the association between PND and adverse infant health outcomes, relative risk estimates obtained from each primary study were collected and meta-analyzed to get a single estimate for each adverse birth outcomes. The meta-analysis for each objective was reported in a separate forest plot, and their main findings were summarized in tables. For studies that lacked adjusted estimates, crude estimates were used. Where a single study reported more than one adverse birth outcomes, pooling was done for each outcome.

\section{Risk of bias and adjustment}

Cochran Q $\left(I^{2}\right)$, visual inspection of forest plot, Galbraith plot [30], and Higgins test [31] were used to assess the presence of heterogeneity. The DerSimonian and Lairds random-effect model was used to pool odds ratio or relative risk estimates in the presence of heterogeneity. These sub-group analyses investigated differences between and within groups' effect. Study setting, study design, year of investigation, tools used for measuring depression, sample size, and income of the country were used as base for sub-analysis. Visual inspection of a funnel plot asymmetry and Egger's regression test were used to check for potential publication bias [32, 33]. The L estimator of Duval and Tweedie was used to find and fill the missed studies through the procedure called the trim-and-fill analysis [34]. A meta-analysis was conducted after log-transforming the estimates from primary studies. As overall adverse infant health outcomes are considered rare in this studies (most of the are close to one), ORs, RRs, and HRs were assumed as a reasonable approximations of each other [35-37]. Sensitivity analysis was also conducted to test for the presence of studies with outlier estimates. All analyses were conducted in Stata 14 [38].

\section{Protocol registration}

This review was registered in PROSPERO with protocol number CRD42017082624.

\section{Result}

In total, 1291 records were retrieved from databases. After removing duplicates, reviewing titles, and abstracts, 149 records were deemed eligible for full text review. After exclusion of 67 records in full text review, 84 records were assessed for quality. Lastly, 58 articles on PND [39-95] and 17 [56, 58, 83, 85, 86, 89-91, 95-103] articles on adverse infant health outcomes were assessed as good quality and were included in quantitative analysis (Fig. 1).

\section{Postnatal depression}

Among 58 studies (with 63,293 population), 47 (81\%) studies were conducted in middle-income countries, $38(66 \%)$ were institutional-based studies, and $41(70 \%)$ used EPDS for screening depression. Fourteen studies were conducted in Africa, 13 studies were from countries located in North and South America, and 31 studies were conducted in Asia. A wide range of PND prevalence $(3.5 \%$ in Ghana to $58.8 \%$ in Iran) was observed across the studies. The studies were published from 2007 to 2017 with sample sizes ranging between 87 and 16,560 participants. (Table 1) Because of substantial evidence of heterogeneity among primary studies as evidenced by Cochran Q $\left(I^{2}=99.0 \%\right)$, visual inspection of forest plot, and Higgins test $(p=0.001)$, estimate from DerSimonian and Lairds random-effect model was reported in a sub-group analysis. Because of evidence in publication bias, an estimate from trim and fill analysis was reported in each sub-analysis (Eggers test, $P<0.01$ ). A sensitivity analysis showed a PND prevalence ranging between 18.82 and $25.02 \%$.

Postnatal depression increased during the last seven years, from $18.2 \%$ (95\%CI: $12.8-23.5 \%$ ) in $2010-2012$ to 25.6\% (95\%CI: $19.9-27.2 \%)$ in 2016-17. The prevalence was higher in low-income countries (Pooled Prevalence $(\mathrm{PP})=25.8 \% ; \quad 95 \% \mathrm{CI}: \quad 17.9-33.8 \%)$ and in health institutional-based studies ( $\mathrm{PP}=22.1 \%$; 95\%CI: 18.8 25.3\%). Postnatal depression increases from the earliest weeks of birth ( $\mathrm{PP}=17.6 \%$; $95 \% \mathrm{CI} ; 7.7-27.5 \%)$ to the end of second year $(\mathrm{PP}=25.2 \%$; 95\%CI: $19.9-30.5 \%)$. Postnatal depression was the highest in studies used Beck Depression Inventory (BDI), Center for Epidemiological Studies Depression scale (CED), the Structured Clinical Interview for DSM-IV Axis I Disorders (SCIDI), Mini International Neuropsychiatric Interview (MINI) 


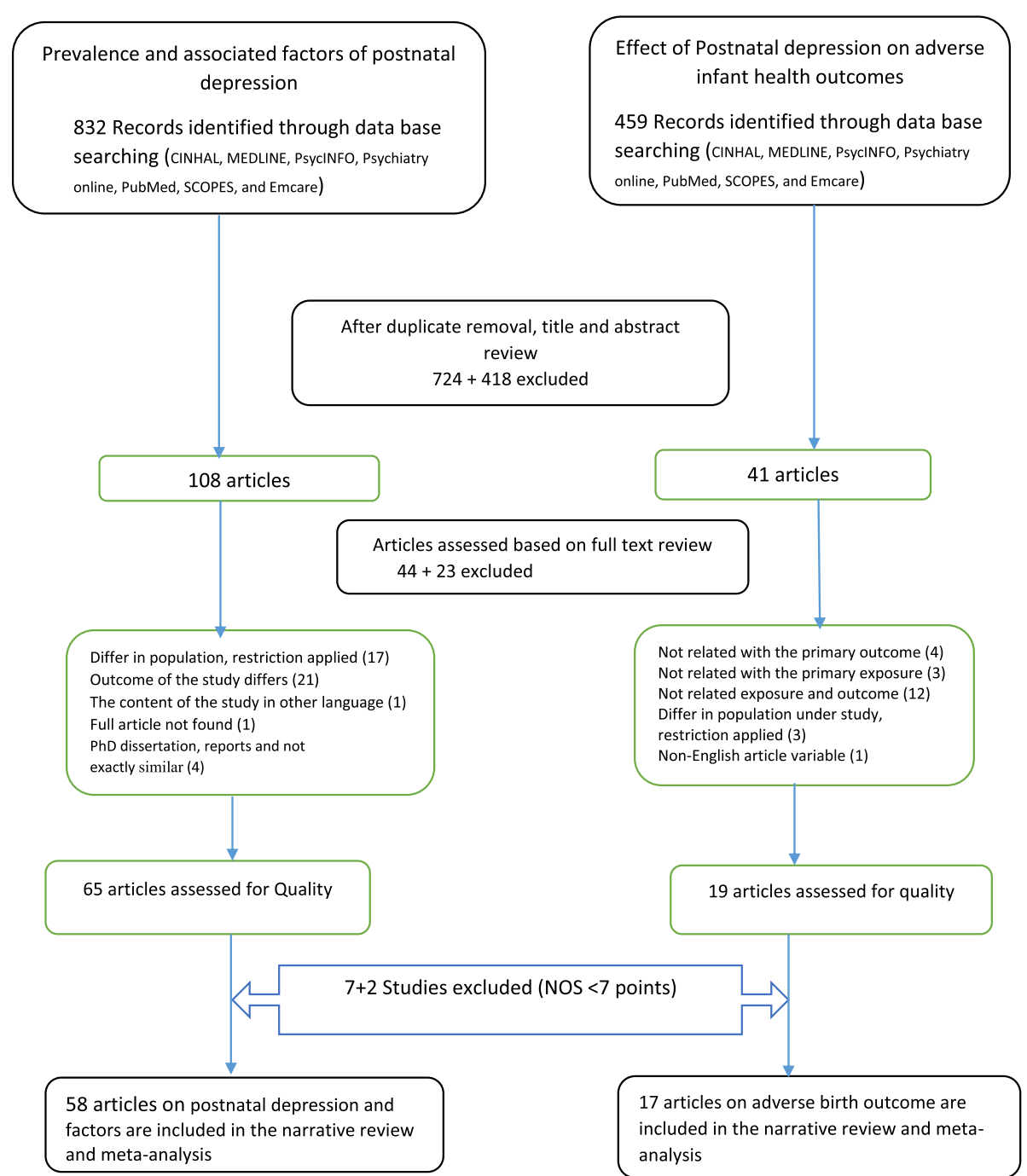

Fig. 1 Flow chart of study inclusion for systematic review and meta-analysis of postnatal depression and its effect on adverse infant health outcomes in low and middle-income countries, 2007-2017

( $\mathrm{PP}=28.3 \% ;$ 95\%CI: 16.9-39.8\%), and in studies with small sample (PP = 23.3\%; 95\%CI: 20.2-26.5\%) (Table 2).

Poor obstetric history (Pooled Odds Ratio (POR) = 1.98; 95\%CI: 1.66-2.36) and social support (POR = 2.44; 95\%CI: 1.92-3.09), exposure to history of CMD (POR = 3.30; 95\%CI: 1.88-5.80) and violence (POR $=2.61$; 95\%CI: 2.16-3.15), low economic $(\mathrm{POR}=2.05 ; 95 \% \mathrm{CI}$ : $1.66-2.54)$ and educational status $(\mathrm{POR}=2.06$; $95 \% \mathrm{CI}$ : 1.56-2.73), and problem with maternal and newborn health $(\mathrm{POR}=3.16$; 95\%CI: $1.96-5.08)$ were risk factors for PND (Table 3).

The association between postnatal depression on adverse infant health outcomes

Seventeen studies (33 estimates), with a total of 31,454 participants, were included in this analysis. Nine studies from Africa, eight from Asia, and four from countries in
North America were found. Fifteen studies represented middle-income countries, and five studies represented low-income countries. Twelve (57\%) studies were longitudinal, 12 (57\%) were community-based, and their sample size ranged from 166 to 16,560 participants. Center for Epidemiological Studies Depression scale (CED) and EPDS screening tools were used in 7 (33\%) and 5 (23.8\%) of the studies, respectively (Table 4 ).

Of 33 estimates, 19 were on malnutrition, 10 were on common infant illness, and 4 were on non-exclusive breastfeeding. The following authors studied more than one outcome in a single study: Adewuya et al. studied two different forms of malnutrition (stunting and underweight); Guo et al. studied febrile illnesses in two countries (Ghana and Côte d'Ivoire); Weobong et al. studied four different types of febrile illnesses (diarrhea, cough, fever, vomiting); Madeghe et al. studied two different 
Table 1 Summary of studies conducted on postnatal depression and associated factors in low and middle-income countries (20072017), $N=58$

\begin{tabular}{|c|c|c|c|c|c|c|c|}
\hline Author, P. year & Year & Country, income & Study setting & Sample size & Time of assessment & Tool used & Prevalence \\
\hline Dindar I et al. 2007 & 2007 & Middle & $\mathrm{HI}$ & 679 & birth to 48 weeks & EPDS & $25.6 \%$ \\
\hline Ege E et al. 2008 & 2008 & Middle & community & 364 & 6 to 48 weeks & EPDS & $33.2 \%$ \\
\hline Flores-Quijano ME et al., 2008 & 2008 & Middle & $\mathrm{HI}$ & 163 & 2 to 12 weeks & EPDS & $24.5 \%$ \\
\hline Hasselmann MH et al., 2008 & 2008 & Middle & $\mathrm{HI}$ & 429 & birth to 8 weeks & EPDS & $35.8 \%$ \\
\hline Tannous L et al., 2008 & 2008 & Middle & community & 271 & 6 to 8 weeks & EPDS & $20.7 \%$ \\
\hline Durat and Kutlu, 2010 & 2009 & Middle & $\mathrm{HI}$ & 126 & birth to 48 weeks & EPDS & $23.8 \%$ \\
\hline Yagmur Y et al., 2010 & 2010 & Middle & community & 730 & birth to 48 weeks & EPDS & $21.0 \%$ \\
\hline Botino M et al., 2012 & 2012 & Middle & $\mathrm{HI}$ & 811 & birth to 20 weeks & EPDS & $24.3 \%$ \\
\hline Goker A et al., 2012 & 2012 & Middle & $\mathrm{HI}$ & 318 & 4 to 48 weeks & EPDS & $31.4 \%$ \\
\hline Pocan Ag et al., 2013 & 2013 & Middle & $\mathrm{HI}$ & 187 & 4 to 6 weeks & EPDS & $28.9 \%$ \\
\hline Melo Jr. EF et al., 2012 & 2012 & Middle & $\mathrm{HI}$ & 555 & 4 to 6 weeks & EPDS & $10.8 \%$ \\
\hline Mathisen SE et al. 2013 & 2013 & Middle & $\mathrm{HI}$ & 87 & 4 to 6 weeks & EPDS & $37.2 \%$ \\
\hline Serhan N et al. 2013 & 2012 & Middle & community & 110 & 8 to 24 weeks & EPDS & $9.1 \%$ \\
\hline de Castro et al 2015 & 2014 & Middle & $\mathrm{HI}$ & 604 & birth to 36 weeks & EPDS & $10.6 \%$ \\
\hline Lara MA et al., 2015 & 2014 & Middle & $\mathrm{HI}$ & 210 & 24 weeks & SCID-I & $13.4 \%$ \\
\hline Corrêa H et al., 2016 & 2016 & Middle & $\mathrm{HI}$ & 3060 & birth to 48 weeks & EPDS & $19.5 \%$ \\
\hline Robert A et al., 2016 & 2016 & Middle & $\mathrm{HI}$ & 194 & birth to 6 weeks & EPDS & $40.2 \%$ \\
\hline Ho-Yen SD et al. 2007 & 2007 & Low & community & 426 & 4 to five weeks & EPDS & $4.9 \%$ \\
\hline Baghianimoghadam et al, 2009 & 2007 & Middle & $\mathrm{HI}$ & 120 & 1 to 16 weeks & $\mathrm{BDI}$ & $58.8 \%$ \\
\hline Gao L et al., 2009 & 2008 & Middle & $\mathrm{HI}$ & 130 & 6 to 8 weeks & EPDS & $13.8 \%$ \\
\hline Kadir AA, et al., 2009 & 2009 & Middle & $\mathrm{HI}$ & 293 & 4 to 6 weeks & EPDS & $27.3 \%$ \\
\hline Wan EY et al., 2009 & 2009 & Middle & $\mathrm{HI}$ & 342 & 6 to 8 weeks & EPDS & $15.5 \%$ \\
\hline Petrosyan D, et al.,2011 & 2011 & Middle & $\mathrm{HI}$ & 437 & 3 months & EPDS & $14.4 \%$ \\
\hline Ahmed HM et al., 2012 & 2012 & Middle & $\mathrm{HI}$ & 1000 & 6 to 8 weeks & EPDS & $28.4 \%$ \\
\hline Hegde S et al. 2012 & 2012 & Middle & $\mathrm{HI}$ & 150 & 6 to 14 weeks & EPDS & $15.5 \%$ \\
\hline Zainal NZ et al. 2012 & 2012 & Middle & $\mathrm{HI}$ & 411 & 6 to 8 weeks & $\mathrm{MINI}$ & $6.8 \%$ \\
\hline Swapan G et al. 2013 & 2013 & Middle & $\mathrm{HI}$ & 202 & 6 weeks & PRIME MD & $15.8 \%$ \\
\hline Panyayong B et al., 2013 & 2013 & Middle & community & 1731 & 6 to 8 weeks & EPDS & $8.4 \%$ \\
\hline Abdollahi F et al., 2014 & 2014 & Middle & $\mathrm{HI}$ & 1801 & 8 to 12 weeks & EPDS & $4.5 \%$ \\
\hline Deng AW et al., 2014 & 2014 & Middle & community & 1823 & 4 weeks & EPDS & $27.4 \%$ \\
\hline El-Hachem C et al. 2014 & 2014 & Middle & $\mathrm{HI}$ & 228 & 4 weeks & EPDS & $12.0 \%$ \\
\hline Giri RK et al. 2015 & 2015 & Low & $\mathrm{HI}$ & 346 & 6 to 10 weeks & EPDS & $30.0 \%$ \\
\hline Yusuff ASM et al. 2015 & 2015 & Middle & $\mathrm{HI}$ & 2072 & 4 to 24 weeks & EPDS & $14.3 \%$ \\
\hline Murray L et al. 2015 & 2015 & Middle & $\mathrm{HI}$ & 431 & 4 to 24 weeks & EPDS & $18.1 \%$ \\
\hline Shivalli S et al. 2015 & 2015 & Middle & $\mathrm{HI}$ & 102 & 4 to 6 weeks & EPDS & $31.4 \%$ \\
\hline Abdollahi et al. 2014 & 2014 & Middle & $\mathrm{HI}$ & 1910 & 12 weeks & EPDS & $19.0 \%$ \\
\hline Safadi RR et al. 2016 & 2016 & Low & $\mathrm{HI}$ & 315 & 12 weeks & PHQ-9 & $25.0 \%$ \\
\hline Iranpour S et al. 2017 & 2017 & Middle & community & 360 & 12 weeks & EPDS & $34.8 \%$ \\
\hline Liu S et al. 2017 & 2017 & Middle & community & 882 & 4 weeks & EPDS & $6.7 \%$ \\
\hline Ramchandani PG et al. 2009 & 2008 & Middle & community & 1035 & 24 weeks & PDQ & $16.4 \%$ \\
\hline Stewart RC et al. 2010 & 2009 & Middle & $\mathrm{HI}$ & 501 & 36 weeks & DSM-IV & $13.9 \%$ \\
\hline Hassanein I et al. 2014 & 2014 & Low & $\mathrm{Hi}$ & 290 & 12 weeks & EPDS & $39.0 \%$ \\
\hline Mohammed ES et al. 2014 & 2014 & Low & community & 200 & 56 weeks & EPDS & $49.5 \%$ \\
\hline
\end{tabular}


Table 1 Summary of studies conducted on postnatal depression and associated factors in low and middle-income countries (20072017), $N=58$ (Continued)

\begin{tabular}{|c|c|c|c|c|c|c|c|}
\hline Author, P. year & Year & Country, income & Study setting & Sample size & Time of assessment & Tool used & Prevalence \\
\hline Khalifa DS et al. 2015 & 2015 & Low & $\mathrm{HI}$ & 300 & 12 weeks & EPDS & $9.2 \%$ \\
\hline Stellenberg E et al. 2015 & 2016 & Middle & community & 159 & 6 to 14 weeks & EPDS & $50.3 \%$ \\
\hline Weobong B et al. 2013 & 2016 & Middle & community & 13,360 & 4 weeks & PHQ-9 & $3.8 \%$ \\
\hline Shamu S et al., 2008 & 2016 & Low & $\mathrm{HI}$ & 842 & 6 weeks & CES-D & $21.4 \%$ \\
\hline Azale A et al. 2016 & 2016 & Low & Community & 385 & 24 weeks & PHQ_9 & $12.1 \%$ \\
\hline Surkan PJ et al. 2009 & 2009 & Middle & $\mathrm{HI}$ & 595 & 24 to 48 weeks & CES-D & $56.0 \%$ \\
\hline Machado MC et al. 2014 & 2014 & Middle & $\mathrm{HI}$ & 168 & 4 t0 12 weeks & EPDS & $16.1 \%$ \\
\hline Gausia K et al. 2010 & 2010 & Low & Community & 318 & 6 to 8 weeks & EPDS & $20.1 \%$ \\
\hline Upadhyay AK et al. 2016 & 2016 & Middle & Community & 1833 & 20 to 84 weeks & SRQ-20 & $29.8 \%$ \\
\hline Islam MJ et al. 2017 & 2016 & Low & Community & 426 & 24 weeks & EPDS & $35.2 \%$ \\
\hline Saeed Q et al. 2016 & 2016 & Low & Community & 325 & 96 weeks & AKUADS & $40.0 \%$ \\
\hline Ndokera R et al. 2008 & 2008 & Middle & community & 278 & 8 to 48 weeks & SRQ-20 & $9.7 \%$ \\
\hline Guo N et al. 2013 (Ghana) & 2013 & Middle & $\mathrm{HI}$ & 654 & 12 weeks & PHQ_9 & $8.9 \%$ \\
\hline Guo N et al. 2013(Ivory Coast) & 2013 & Middle & $\mathrm{HI}$ & 654 & 12 weeks & PHQ_9 & $11.8 \%$ \\
\hline Weobong B et al. 2015 & 2017 & Middle & Community & 16,560 & 4 to 12 weeks & DSM-IV & $3.5 \%$ \\
\hline
\end{tabular}

HI health institution, PDQ Pitt depression questionnaire, AKUADS Aga Khan University Anxiety and Depression Scale> = 20, EPDS Edinburgh Postnatal Depression Scale, $B D I$ Beck depression inventory, CED Center for Epidemiological Studies Depression scale, SCID-I Structured Clinical Interview for the DSM-IV depression module, MINI Mini International Neuropsychiatric Interview, PHQ-9 patient health questioner, SRQ-20 Self Reporting Questionnaire

forms of outcome (Non-exclusive breast feeding and underweight); Ndokera et al. studied three different forms of outcomes (serios illnesses, diarrhea, underweight); Benett et al. studied two different forms of malnutrition in four countries (stunting and underweight in India, Ethiopia, Peru, and Vietnam) (Table 4) All type of outcomes were sub-analyzed based on the three major forms of adverse infant health outcomes as stated in the Integrated Management of Newborn and Childhood Illnesses (IMNCI) guideline [104]: febrile illnesses (accounts for fever, diarrhea, vomiting, cough); malnutrition (underweight, stunting, malnutrition); and non-exclusive breastfeeding (Fig. 2).

Seventeen estimates were reported in relative risk (RR), 13 in odds ratio (OR), while the rest three estimated the hazard ratio (HR). The association between PND and malnutrition (underweight, wasting, stunting, short stature), common infant illness (diarrhea, febrile illnesses, cough), and non-exclusive breastfeeding were significant in 12, eight, and three studies, respectively. As a small study effect and high heterogeneity were evidenced, the final estimate corrected for trim and fill analyses from a random effect model was reported (Figs. 3 and 4). Accordingly, PND was associated with 1.31 times increased risk of adverse infant health outcomes (95\%CI: 1.17-1.48). The sub-analyses based on the types of outcomes showed the following: a risk of being malnourished, being sick by common infant illnesses, and having non-exclusively breastfeeding was 1.39 times (95\%CI: 1.21-1.61), 1.55 times (95\%CI; 1.39-1.74), and 2.55 times (95\%CI; $1.41-$
4.61) higher among infants of depressed than nondepressed mothers, respectively (Fig. 2).

A sub-analysis was conducted to explore the consistency of the association across different characteristics of the studies. Accordingly, (1) a pooled estimate from the OR (Pooled Odds Ratio $(\mathrm{POR})=2.62$; $95 \% \mathrm{CI}$ : 2.03-3.38) was larger than the $R R(P R R=1.24 ; 95 \% C I$ : $1.10-1.41)$ and the $\mathrm{HR}(\mathrm{PHR}=1.44 ; 95 \% \mathrm{CI}: 1.21-1.70)$; (2) the risk was similar for studies used screening (EPDS, PHQ, SRQ) and diagnostic tool (DSM/MINI); (3) the risk of adverse infant health outcomes decreased as age of the infant increased; from 1.75 (95\%CI; 1.51-2.03) at the age of 0 to 6 months to 1.28 (95\%CI: $1.06-1.54$ ) at the age of 12 months and above; (4) the risk of adverse infant health outcomes was lower in low-income countries $(\mathrm{PRR}=1.40 ; 95 \% \mathrm{CI}: 1.37-1.74)$ compared to middleincome countries ( $\mathrm{PRR}=1.59 ; 95 \% \mathrm{CI}$ : 1.40-1.81); and (5) as sample size increased the association between PND and adverse infant health outcome decreased; from 1.98 (95\%CI; 1.63-2.40) for those included a sample size less than 1500 to 1.27 (95\%CI; 1.10-1.46) for the studies with larger sample sizes. (Supplementary information) According to the sensitivity analysis, the pooled relative risks ratio was not affected when individual studies were omitted (Fig. 5).

\section{Discussion}

The current comprehensive and relatively sizeable review and meta-analyses have dealt with the burden, risk factors, and effects of PND on infant health outcomes in 
Table 2 Sub-analysis of postnatal depression prevalence in low and middle-income countries ( $N=58$, 2007-2017), (random effect model, result after a trim and fill analysis)

\begin{tabular}{|c|c|c|c|}
\hline Variable of sub-analysis & Number of studies & Sample size & Pooled prevalence; $95 \% \mathrm{Cl}$ \\
\hline \multicolumn{4}{|l|}{ Year of publication } \\
\hline 2007-2009 & 15 & 5752 & $25.1(18.1-32.2)$ \\
\hline 2010-2012 & 10 & 4840 & $18.2(12.8-23.5)$ \\
\hline 2013-2015 & 20 & 14,000 & $19.6(15.8-23.5)$ \\
\hline 2016-2017 (two years) & 13 & 38,701 & $25.6(19.9-27.2)$ \\
\hline \multicolumn{4}{|l|}{ Income of the country } \\
\hline Low income & 11 & 4173 & $25.8(17.9-33.8)$ \\
\hline Middle income & 47 & 59,120 & $20.7(18.4-23.1)$ \\
\hline \multicolumn{4}{|l|}{ Study setting } \\
\hline Health institution & 38 & 21,717 & $22.1(18.8-25.3)$ \\
\hline Community based & 20 & 41,576 & $20.9(17.9,23.9)$ \\
\hline \multicolumn{4}{|l|}{ Time of screening } \\
\hline Birth to four weeks & 5 & 16,487 & $17.6(7.8-27.5)$ \\
\hline 5 weeks to 10 weeks & 22 & 26,599 & $21.9(18.0-25.7)$ \\
\hline 11 weeks to 16 weeks & 12 & 7683 & $17.9(14.1-21.8)$ \\
\hline 17 weeks to 96 weeks & 19 & 12,524 & $25.2(19.9-30.5)$ \\
\hline \multicolumn{4}{|c|}{ A tool used for depression screening } \\
\hline EPDS & 41 & 25,013 & $22.6(19.6,25.7)$ \\
\hline PHQ-9 and SRQ-20 & 7 & 17,479 & $14.4(6.2-22.6)$ \\
\hline DSM-IV & 2 & 17,061 & $8.6(1.6-18.8)$ \\
\hline Other/BDI, CED, SCID-I, MINI/ & 8 & 3740 & $28.3(16.9-39.8)$ \\
\hline \multicolumn{4}{|l|}{ Sample size } \\
\hline$<=1091$ & 49 & 19,143 & $23.4(20.2-26.5)$ \\
\hline$>1091$ & 9 & 44,150 & $14.4(10.6-18.1)$ \\
\hline
\end{tabular}

EPDS Edinburgh Postnatal Depression Scale, BDI Beck depression inventory, CED Center for Epidemiological Studies Depression scale, SCID-I structured clinical interview for the DSM-IV depression module, MINI Mini International Neuropsychiatric Interview, PHQ-9 patient health questioner, SRQ-20 self reporting questionnaire

Table 3 Summary of risk factors significantly associated with postnatal depression $(N=58,2007-2017)$, (random effect model, result after a trim and fill analysis)

\begin{tabular}{|c|c|c|c|c|}
\hline Variable of sub-analysis & $\begin{array}{l}\text { Number of } \\
\text { studies }\end{array}$ & $\begin{array}{l}\text { Sample } \\
\text { size }\end{array}$ & $\begin{array}{l}\text { Pooled Odds Ratio (POR), } \\
95 \% \mathrm{Cl}\end{array}$ & $\begin{array}{l}I^{2}, \\
p \text {-value }\end{array}$ \\
\hline $\begin{array}{l}\text { Poor obstetric history (unplanned pregnancy, GDM, GHP, labor complication, history } \\
\text { of emesis, multiparty) }\end{array}$ & 18 & 28,766 & $1.98(1.66-2.36)$ & $\begin{array}{l}64.5 \% \\
p=0.001\end{array}$ \\
\hline $\begin{array}{l}\text { History of CMD (depression during pregnancy, family psychiatric illness, stressful life } \\
\text { event) }\end{array}$ & 13 & 10,074 & $3.30(1.88-5.80)$ & $\begin{array}{l}99.2 \% \\
p=0.001\end{array}$ \\
\hline Poor social support & 12 & 11,206 & $2.44(1.92,3.09)$ & $\begin{array}{l}73.8 \% \\
p=0.001\end{array}$ \\
\hline Low economic status & 12 & 7671 & $2.05(1.66-2.54)$ & $\begin{array}{l}96.2 \% \\
p=0.001\end{array}$ \\
\hline Problem with maternal and newborn health & 11 & 5954 & $3.16(1.96-5.08)$ & $\begin{array}{l}91.7 \% \\
p=0.001\end{array}$ \\
\hline Exposure to any forms of violence (physical, emotional, sexual) & 7 & 5730 & $2.61(2.16-3.15)$ & $\begin{array}{l}0 \% \\
p=0.867\end{array}$ \\
\hline Low educational status of the mother & 7 & 5549 & $2.06(1.56-2.73)$ & $\begin{array}{l}48.2 \% \\
P=0.07\end{array}$ \\
\hline
\end{tabular}


Table 4 Summary of studies conducted on the effect of postnatal depression on infant health outcomes in low and middle-income countries, 2007-2017 ( $N=14)$

\begin{tabular}{|c|c|c|c|c|c|c|c|c|c|c|}
\hline Author, P. year & $\begin{array}{l}\text { Country, } \\
\text { income }\end{array}$ & $\begin{array}{l}\text { Study } \\
\text { setting }\end{array}$ & $\begin{array}{l}\text { Study } \\
\text { design }\end{array}$ & $\begin{array}{l}\text { Sample } \\
\text { size }\end{array}$ & weeks & $\begin{array}{l}\text { Screening } \\
\text { tool used }\end{array}$ & $\begin{array}{l}\text { Infant adverse } \\
\text { health outcomes }\end{array}$ & $\begin{array}{l}\text { Estimate } \\
\text { (RR/OR) }\end{array}$ & $\mathrm{LCl}$ & $\mathrm{UCl}$ \\
\hline $\begin{array}{l}\text { Hasselmann MH et al. } \\
2008\end{array}$ & Brazil, Middle & $\mathrm{HI}$ & cohort & 429 & 4 to 8 weeks & EPDS & Non-EB & 1.21 & 1.02 & 1.45 \\
\hline Surkan PJ et al. 2008 & Brazil, Middle & $\mathrm{HI}$ & $\begin{array}{l}\text { cross } \\
\text { sectional }\end{array}$ & 595 & $\begin{array}{l}6 \text { to } 12 \\
\text { months }\end{array}$ & CES-D & Short stature & 1.8 & 1.1 & 2.9 \\
\hline Machado MC et al. 2014 & Brazil, Middle & $\mathrm{HI}$ & longitudinal & 168 & $\begin{array}{l}1 \text { to } 3 \\
\text { months }\end{array}$ & EPDS & Non-EB & 1.61 & 1.19 & 2.19 \\
\hline Gausia K et al. 2010 & Bangladish, Low & Community & longitudinal & 318 & 6 to 8 weeks & EPDS & Diarrhea & 1.74 & 1.25 & 3.42 \\
\hline Rahman A et al. 2016 & Pakistan, Low & Community & cohort & 279 & 6 months & DSM-IV & Non-EB & 1.42 & 0.98 & 2.06 \\
\hline Upadhyay AK et al. 2016 & India, Middle & Community & longitudinal & 1833 & $\begin{array}{l}5 \text { to } 21 \\
\text { months }\end{array}$ & SRQ-20 & Stunting & 1.53 & 1.21 & 1.92 \\
\hline Islam MJ et al. 2017 & Bangladish, Low & Community & $\begin{array}{l}\text { cross } \\
\text { sectional }\end{array}$ & 426 & 6 months & EPDS & Non-EB & 5 & 2.27 & 11.11 \\
\hline \multirow[t]{2}{*}{ Saeed Q et al. 2016} & \multirow[t]{2}{*}{ Pakistan, Low } & \multirow[t]{2}{*}{ Community } & \multirow{2}{*}{$\begin{array}{l}\text { cross } \\
\text { sectional }\end{array}$} & \multirow[t]{2}{*}{325} & \multirow[t]{2}{*}{2 years } & \multirow[t]{2}{*}{ AKUAD } & Stunting & 3.15 & 1.91 & 5.18 \\
\hline & & & & & & & Underweight & 3.26 & 1.99 & 5.34 \\
\hline \multirow[t]{2}{*}{ Adewuya AO et al. 2007} & \multirow[t]{2}{*}{ Nigeria, Middle } & \multirow[t]{2}{*}{$\mathrm{HI}$} & \multirow[t]{2}{*}{ case control } & \multirow[t]{2}{*}{242} & \multirow{2}{*}{$\begin{array}{l}6 \text { to } 12 \\
\text { weeks }\end{array}$} & \multirow[t]{2}{*}{ SCID-NP } & Poor weight & 3.41 & 1.3 & 8.52 \\
\hline & & & & & & & Poor height & 3.28 & 1.03 & 10.47 \\
\hline Guo N et al. 2013 & Ghana, Middle & $\mathrm{HI}$ & Longitudinal & 654 & 3 months & $\mathrm{PHQ}$ & Febrile illnesses & 1.32 & 1.01 & 1.74 \\
\hline Guo N et al. 2013 & $\begin{array}{l}\text { Côte d'Ivoire } \\
\text { Middle }\end{array}$ & & & & & & Febrile illnesses & 1.57 & 1.20 & 2.07 \\
\hline Ashaba Set al 2013 & Uganda, Low & $\mathrm{HI}$ & Case control & 166 & $1-5 y r s$ & (M.I.N.I.) & Malnutrition & 2.4 & 1.11 & 5.18 \\
\hline \multirow[t]{4}{*}{ Weobong B et al. 2017} & \multirow[t]{4}{*}{ Ghana, Middle } & \multirow[t]{4}{*}{ Community } & \multirow[t]{4}{*}{ Longitudinal } & \multirow[t]{4}{*}{16,560} & \multirow{4}{*}{$\begin{array}{l}4 \text { to } 12 \\
\text { weeks }\end{array}$} & \multirow[t]{4}{*}{ DSM-IV } & Diarrhea & 1.8 & 1.45 & 2.14 \\
\hline & & & & & & & cough & 1.49 & 1.28 & 1.7 \\
\hline & & & & & & & Fever & 1.8 & 1.49 & 2.11 \\
\hline & & & & & & & Vomiting & 1.98 & 1.26 & 2.71 \\
\hline \multirow[t]{2}{*}{ Madeghe BA et al. 2016} & \multirow[t]{2}{*}{ Kenya, Middle } & \multirow[t]{2}{*}{$\mathrm{HI}$} & \multirow{2}{*}{$\begin{array}{l}\text { Cross- } \\
\text { sectional }\end{array}$} & \multirow[t]{2}{*}{200} & \multirow{2}{*}{$\begin{array}{l}6 \text { to } 14 \\
\text { weeks }\end{array}$} & EPDS & Non-EB & 6.14 & 2.45 & 13.36 \\
\hline & & & & & & & Underweight & 4.4 & 1.91 & 11.93 \\
\hline Wemakor A et al. 2016 & Ghana, Middle & $\mathrm{HI}$ & $\begin{array}{l}\text { Cross- } \\
\text { sectional }\end{array}$ & 384 & $\begin{array}{l}0-59 \\
\text { months }\end{array}$ & CED-S & Stunting & 2.48 & 1.29 & 4.77 \\
\hline Ndokera R et al. 2008 & Zambia, Middle & community & Cross- & 278 & $2-12$ & CED & serious illness & 1.64 & 0.51 & 5.24 \\
\hline & & & & & & & diarrhea & 1.32 & 0.71 & 2.48 \\
\hline & & & & & & & underweight & 1.48 & 0.35 & 6.22 \\
\hline $\begin{array}{l}\text { Maureen M Black et al. } \\
2009\end{array}$ & Bangladish, Low & Community & $\begin{array}{l}\text { cross } \\
\text { sectional }\end{array}$ & 221 & $\begin{array}{l}6-12 \\
\text { months }\end{array}$ & CED & Stunting & 2.17 & 1.24 & 3.81 \\
\hline Benett IM et al. 2015 & India, Middle & community & Longitudinal & 1930 & 12 months & CED & Stunting & 1.18 & 1.03 & 1.35 \\
\hline & & & & & & & Underweight & 1.11 & 0.97 & 1.26 \\
\hline & Ethiopia, Low & & & 1885 & & & Stunting & 0.91 & 0.81 & 1.02 \\
\hline & & & & & & & Underweight & 1.01 & 0.89 & 1.15 \\
\hline & Peru, Low & & & 1946 & & & Stunting & 1.06 & 0.93 & 1.22 \\
\hline & & & & & & & underweight & 0.85 & 0.6 & 1.19 \\
\hline & Vietnam Low & & & 1961 & & & Stunting & 0.91 & 0.81 & 1.02 \\
\hline & & & & & & & Underweight & 1.29 & 1.03 & 1.62 \\
\hline
\end{tabular}

low and middle-income countries. The review has included 58 primary studies on PND and 17 studies (with 33 estimates) investigating on PND effect on infant health outcomes in the past ten years. The review founds that a significant number of postnatal mothers were depressed, and their infants suffered from malnutrition, common infant illnesses, and non-exclusive breastfeeding. 


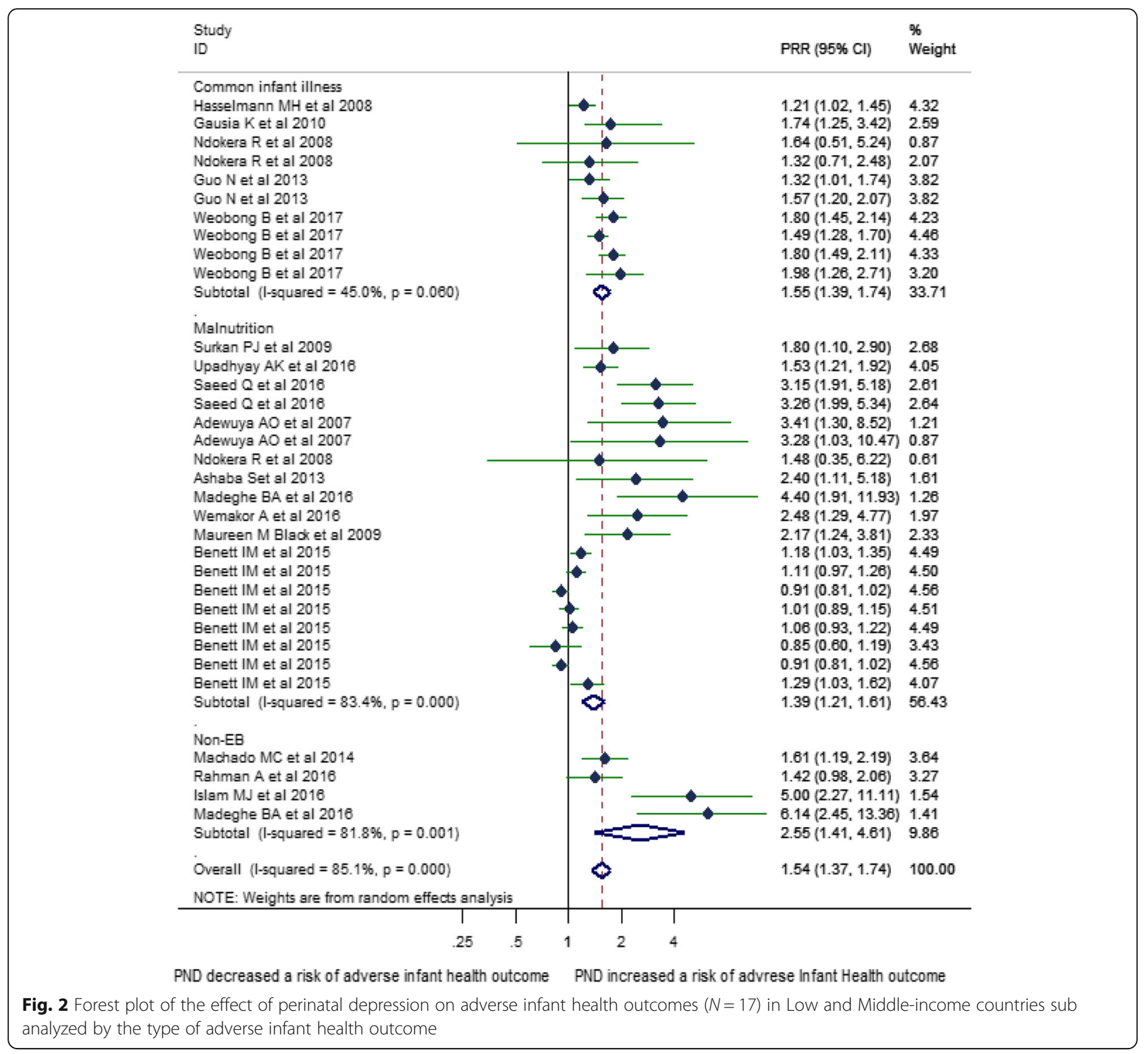

\section{Postnatal depression and its predictors}

The research evidence dealing with PND in low and middle-income countries has been increasing over time. The current meta-analysis showed that one in four and one in five postnatal mothers were living with PND in low-and middle-income countries, respectively. These findings were consistent with a review conducted by Gelaye et al. in low and middle-income countries [12] and slightly higher than a review published by Sawyer et al. [25]. We hypothesized that the rapid decline in reproductive hormones such as estrogen following childbirth might contribute to a dysregulation of the stress hormone, monoamine, and reproductive hormone, which subsequently leads to depression [105]. Postnatal depression prevalence has been increasing from 18 to $25 \%$ in the last seven years, which supports the WHO prediction that depression will be the third global leading cause of morbidity by 2030 [106, 107].

The PND prevalence increased in the first 10 weeks, slightly decreased from 11 to 16 weeks, and steadily rose from 17 to 96 weeks after birth. The trend for the first 16 weeks is similar to a study conducted in developed countries [14, 108]. However, the interpretation for these estimates should account for the window of measurement, as a wider window predicts larger estimates. Community-based studies, studies used diagnostic tools (DSM-IV) for identifying depression, and studies with larger samples predicted relatively prevalence. However, the estimates were consistent across all the sub-analysis that showed the high public health importance of PND in low and middle-income countries. 


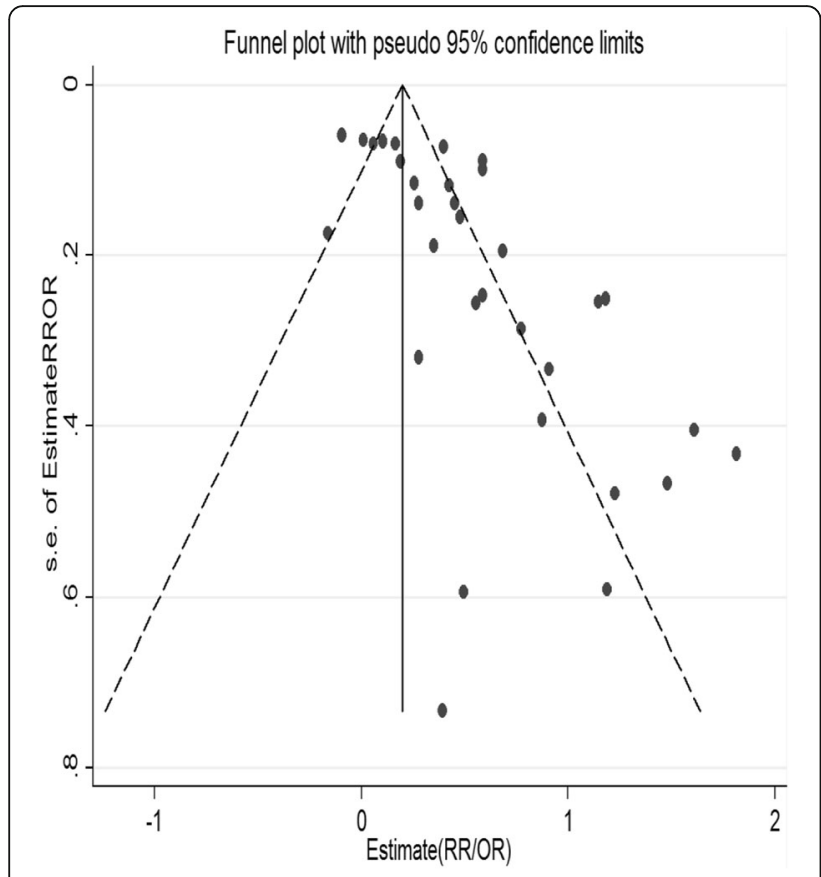

Fig. 3 Funnel plot before Tweedie's and Duval's trim and fill alanysis for testing publication bias

This review identified the following PND predictors: poor obstetric history and social support, history of common mental disorder and violence, low economic and educational status, and maternal and newborn ill health.
Poor obstetric history (unwanted or unplanned pregnancy, multi-parity, history of emesis) strongly predicted PND. These events could be related to household income, mother's un-satisfactory birth experiences, predisposition to bad parenting experience, and post-traumatic stress [23, 25]. Unwanted or unplanned pregnancies could also affect emotional and instrumental support that the mother could get from her partner and related families [109]. Psychosocial factors such as a history of common mental health disorders, poor social support, and exposure to violence, were identified as significant predictors of PND. These findings are consistent with previously published systematic reviews $[12,14,25,110]$. Poor social support could aggravate the mother's stress and depression symptoms as it affects mother's selfconfidence and efficacy [109, 111]. Pregnant mothers who had depression or a history of mental disorders are more likely to develop PND as they are more likely to dampen their positive affect [112], practice rumination and develop a negative cognitive style that could persist throughout the continuum of pregnancy $[113,114]$. Moreover, emerging brain neuroimaging explanations of altered neurocognitive functioning have predicted mother's exposure to childhood abuse or any type of violence as a risk factor for future psychiatric symptomatology $[115,116]$.

Low economic status and a problem with maternal and newborn health were also predicted PND. Mothers living in low-income countries are less likely to access adequate housing, health service, nutrition, and they experienced

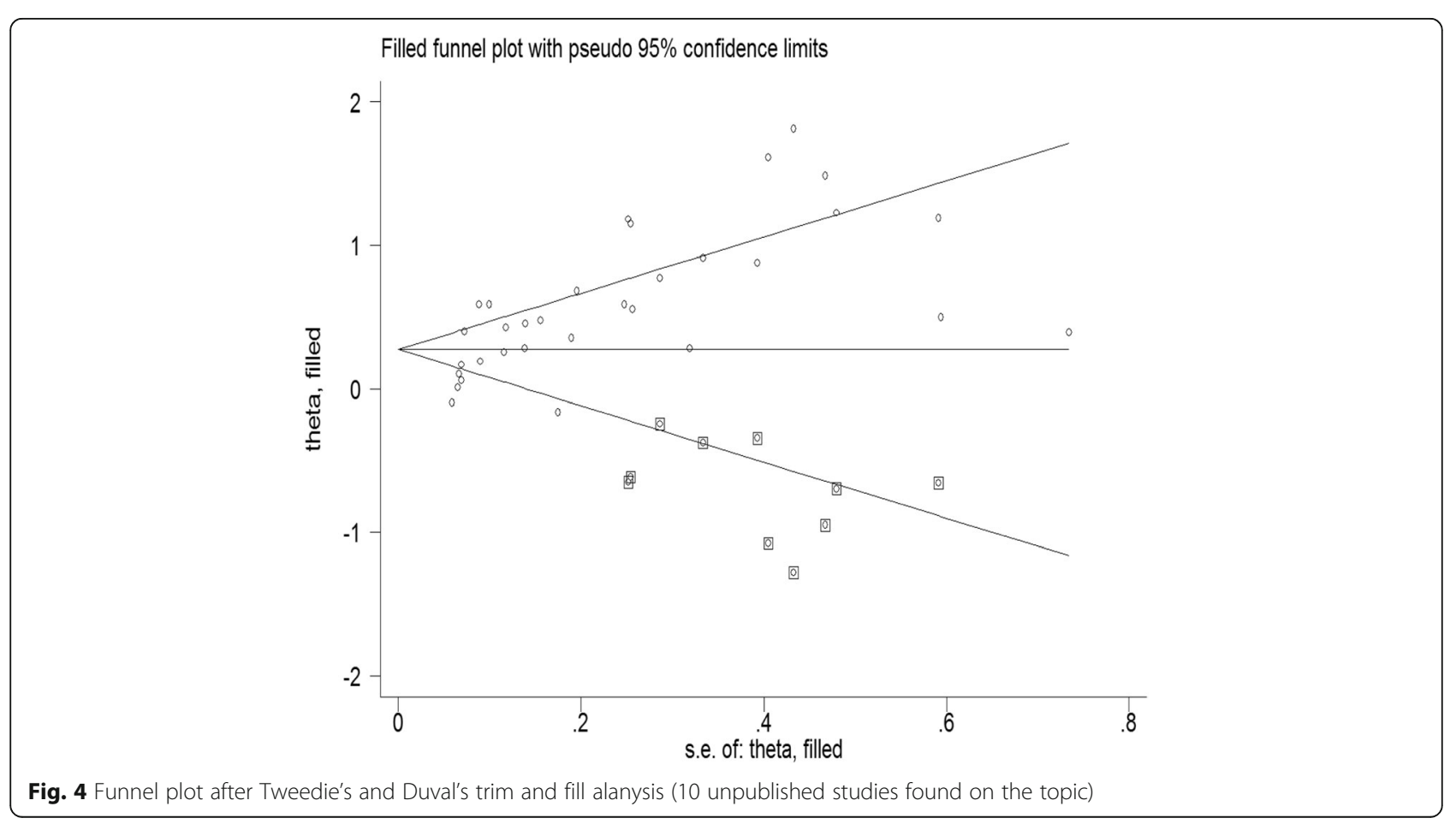




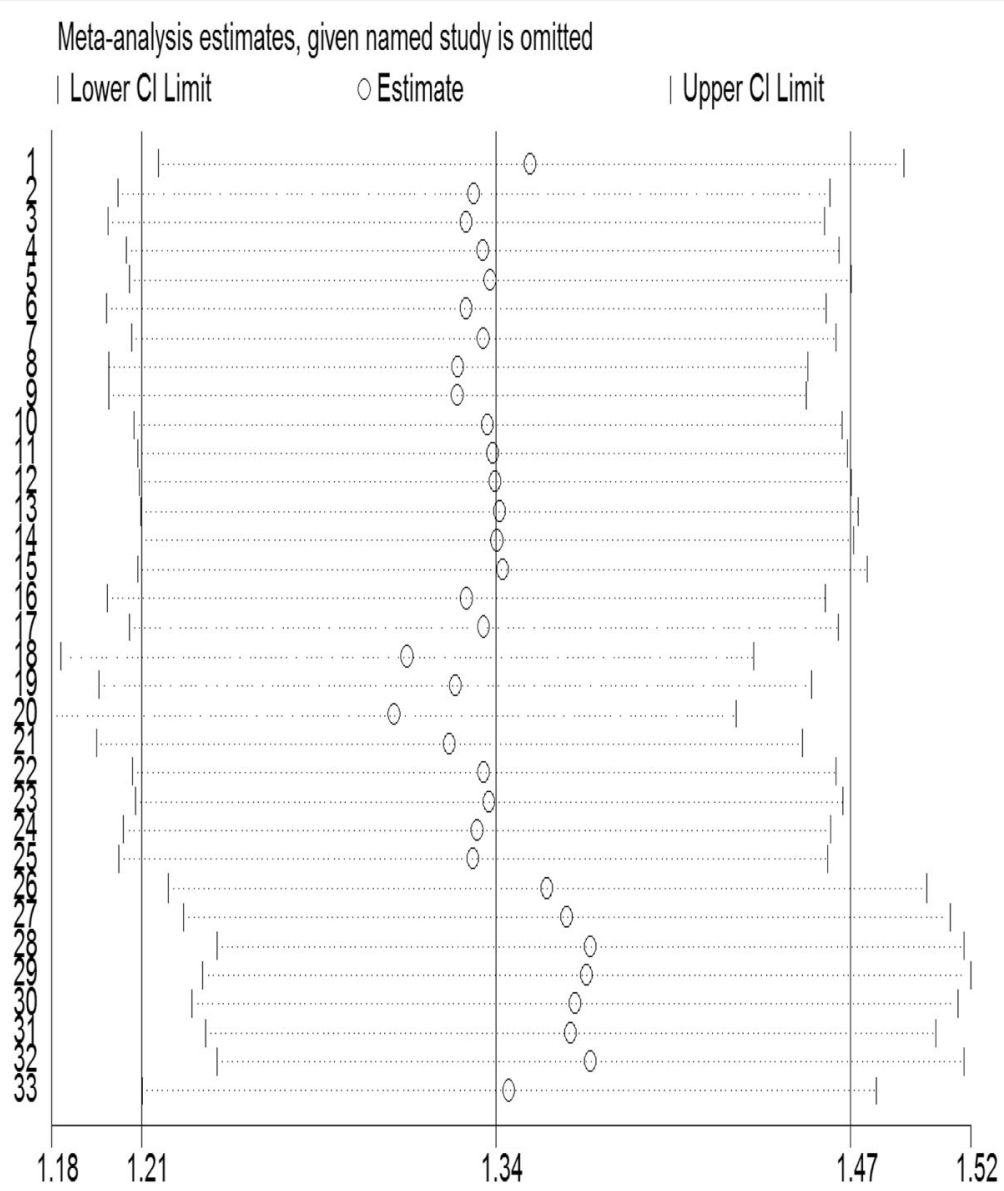

Fig. 5 Sensitivity analysis for estimates on postnatal depression and its effect on adverse infant health outcomes in Low-and Middle-income countries (Number of estimates $=33$ )

challenges in providing adequate care for their infant situations that would add a further layer of stress [11, 117]. Problem with the mother's mental health affects motherinfant interactions, hygienic practices during food preparation and storage, and proper care for the newborn, altogether makes the mothers feel guilty and worthless, subsequently leading to depression $[91,118]$.

\section{Postnatal depression and its effect on adverse infant health outcomes}

Postnatal depression in low-and middle-income countries remains mostly untreated, and there is growing evidence that untreated PND results in adverse infant health outcomes [12, 20-22]. The current systematic review and meta-analysis highlighted the effect of PND on infant health and growth. Postnatal depression increased the risk of adverse infant health outcomes by $31 \%$. PND increased the risk of malnutrition (stunting, wasting, short stature), common infant illnesses, and nonexclusive breastfeeding by $39,55 \%$ and by one and a half fold, respectively. We have estimated that $23.66 \%$ (7442) of postnatal mothers who had their infants suffered from adverse infant health outcomes in the study population $(31,454)$ were attributed because of their depression status and could be averted if depression during the postnatal period would have been treated.

PND as a risk of adverse infant health outcomes was consistent across all type of association measurement, between use of screening tools (EPDS, PHQ, and SRQ) and clinically diagnosed depression (DSM and MINI), in both institutional and community-based studies, in both high- and low-income countries, and irrespective of study sample. Besides, the association between PND and adverse infant health outcomes was not changed across the age of the infant, even though the risk of adverse infant health outcomes decreased as the age of the infant increased.

The association between malnutrition [20, 95], infant morbidity [119] and PND was supported by a similar systematic review and meta-analysis [120]. Three pathways have been proposed to explain the link between mother's PND symptoms and adverse infant health outcomes: (1) genetic, (2) hormonal, neuro-regulatory system impairment, and (3) environmental, an indirect 
effect of PND on the quality of mother's caregiving [20, 121]. The endocrine dysregulation because of PND would compromise a psychosocial functioning that affects a mother-infant interaction [122]. In middle and low-income countries, mothers are more responsible for caring, feeding, and nurturing their newborns than fathers [123] though they are suffering from lack of adequate income, access to quality water, poor sanitation, and knowledge of illnesses and their prevention [13]. Under these highlighted conditions and exacerbated depression symptoms, postnatal mothers are unable to provide the expected level of care to their infant that would affect their growth and wellbeing.

The current finding related to the effect of PND on non-exclusive breastfeeding is consistent with two systematic reviews [124, 125]. Postnatal depression affects the self-efficacy of the mother and their intention to breastfeed, as explained in the breastfeeding self-efficacy theory [126, 127]. Mothers of better self-efficacy initiate breastfeeding early, stay breastfeeding for a longer time, and produce self-encouraging thoughts to easily overcome challenges of breastfeeding.

\section{Strength and limitations}

The major strengths of the current review include: (1) it was up-to-date, means included primary studies published up to December 30, 2017; (2) inclusion of 75 studies $(22$ more studies than the recent systematic review by Gelaye et al. [12]; (3) an in-depth analysis and presentation of PND predictors and; (4) an in-depth and up-to-date estimation and presentation of PND effects on adverse infant health outcomes. However, the estimation would be affected by the type and time of depression measurement, methodological and cultural heterogeneities though we treated this heterogeneity with analytical applications.

Considering the strengths mentioned above and limitations of our systematic review and meta-analysis, we believed in providing the most accurate quantification of PND prevalence, its prominent risk factors and association with infant morbidity, malnutrition, and early breastfeeding cessation in low-and-middle income countries. These findings add one step forward to the consistency of the evidence that PND is a significant public health threat for the birthing mothers and their infants.

\section{Conclusions}

The findings indicate a quarter and one in five postnatal mothers were depressed in low-and middle-income countries, respectively, an indication that it is highly prevalent in the regions. Postnatal mothers with poor obstetric history and social support, history of common mental disorder and childhood violence, low economic status, a problem with maternal and newborn health were more likely to have depression. Postnatal mothers with depression were also at higher risk of having sick, malnourished, and non-exclusively breastfed infant relative to mothers who did not have depression symptoms. More importantly, this effect was similar between studies that clinically diagnosed depression and used selfreporting scales. Based on the current findings, an early screening of postnatal mothers starting from the first four weeks of birth and taking prompt intervention would save the mother and her infant from morbidity, mortality, disability, and future developmental consequences.

\section{Supplementary information}

Supplementary information accompanies this paper at https://doi.org/10. 1186/s12884-020-03092-7.

Additional file 1 : Supplementary material 1: Postnatal

supplementary information.

\begin{abstract}
Abbreviations
CES-D 20: Center for Epidemiologic Studies depression scale 20; DM: Diabetic mellitus; DSM-IV: Diagnostic and statistical manual of mental disorders version 4; EPDS: Edinburgh postnatal depression scale; HIV: Human immune deficiency); IPV: Intimate partner violence; LAMICS: Low and middle-income countries; NOS: New Castle Ottawa Scale; OR: Odds ratio; PDQ: Pitt depression questionnaire; PHQ-9: Patient health questioner-9; PND: Postnatal depression; POR: Pooled odds ratio; PRISMA: Preferred reporting items for systematic review and meta-analysis; RCT: Randomized control trial; RR: Relative risk; SRQ-20: Self reporting questioner; WHO: World Health Organization
\end{abstract}

\section{Acknowledgments \\ Our heartfelt gratitude would go to Mr. Berihun Assefa Dachew for his contribution to quality assessment of the included studies.}

\section{Authors' contributions}

AFD, LM, EMM: conceived the design and developed the search strategy: AFD: searched, screened, and appraised the studies and extract the data: AFD analyzed the data and drafted the manuscript. LM and EMM critically reviewed and revised the manuscript, and all authors read and approved the final manuscript for publication.

\section{Funding}

This research received no specific grant from any funding agency in public, commercial, or not-for-profit sectors.

\section{Availability of data and materials}

All data generated or analyzed during this review are included in this manuscript and its supplementary information files.

Ethics approval and consent to participate Not applicable.

Consent for publication

Not applicable.

Competing interests

None to declare. 
Received: 30 December 2019 Accepted: 3 July 2020 Published online: 22 July 2020

\section{References}

1. WHO. DEPRESSION A Global Public Health Concern 2012.

2. O'Hara MW. Postpartum depression: what we know. J Clin Psychol. 2009; 65(12):1258-69.

3. Beck CT. Postpartum depression: it isn't just the blues. Am J Nurs. 2006; 106(5):40-50 quiz -1.

4. Robertson E, Grace S, Wallington T, Stewart DE. Antenatal risk factors for postpartum depression: a synthesis of recent literature. Gen Hosp Psychiatry. 2004;26(4):289-95

5. O'Hara MW, Wisner KL. Perinatal mental illness: definition, description and aetiology. Best Pract Res Clin Obstetr Gynaecol. 2014;28(1):3-12.

6. Wisner KL, Moses-Kolko EL, Sit DKY. Postpartum depression: a disorder in search of a definition. Arch Womens Ment Health. 2010;13(1):37-40.

7. El-Ibiary SY, Hamilton SP, Abel R, Erdman CA, Robertson PA, Finley PR. A pilot study evaluating genetic and environmental factors for postpartum depression. Innov Clin Neurosci. 2013;10(9-10):15-22.

8. Stern G, Kruckman L. Multi-disciplinary perspectives on post-partum depression: an anthropological critique. Soc Sci Med. 1983;17(15):1027-41.

9. Lund C, Breen A, Flisher AJ, Kakuma R, Corrigall J, Joska JA, et al. Poverty and common mental disorders in low and middle income countries: a systematic review. Soc Sci Med. 2010;71(3):517-28.

10. Beck CT. Predictors of postpartum depression: an update. Nurs Res. 2001; 50(5):275-85.

11. Coast E, Leone T, Hirose A, Jones E. Poverty and postnatal depression: a systematic mapping of the evidence from low and lower middle income countries. Health Place. 2012;18(5):1188-97.

12. Gelaye B, Rondon MB, Araya R, Williams MA. Epidemiology of maternal depression, risk factors, and child outcomes in low-income and middleincome countries. Lancet Psychiatry. 2016;3(10):973-82.

13. Parsons CE, Young KS, Rochat TJ, Kringelbach ML, Stein A. Postnatal depression and its effects on child development: a review of evidence from low- and middle-income countries. Br Med Bull. 2012;101(1):57-79.

14. Beck CT. State of the science on postpartum depression: what nurse researchers have contributed-part 2. MCN Am J Matern Child Nurs. 2008;33: $151 \mathrm{e} 156$.

15. Post and Antenatal Depression Association (PANDA) and Deloitte Access Economics. The cost of perinatal depression in Australia: Final report. Kingston ACT: Deloitte Access Economics; 2012

16. Bodhare TN, Sethi P, Bele SD, Gayatri D, Vivekanand A. Postnatal quality of life, depressive symptoms, and social support among women in southern India. Women Health. 2015;55(3):353-65.

17. Field T. Postpartum depression effects on early interactions, parenting, and safety practices: a review. Infant Behav Dev. 2010;33(1):1-6.

18. Lee DTS, Chung TKH. Postnatal depression: an update. Best Pract Res Clin Obstetr Gynaecol. 2007;21(2):183-91.

19. Feldman R, Granat A, Pariente C, Kanety H, Kuint J, Gilboa-Schechtman E. Maternal depression and anxiety across the postpartum year and infant social engagement, fear regulation, and stress reactivity. J Am Acad Child Adolesc Psychiatry. 2009:48(9):919-27.

20. Surkan PJ, Kennedy CE, Hurley KM, Black MM. Maternal depression and early childhood growth in developing countries: systematic review and metaanalysis. Bull World Health Organ. 2011;89(8):607-15.

21. Understanding Mental IIIness; A Guide to Brain Disorders, Medication, and Therapy, 2011.

22. Grigoriadis S, VonderPorten EH, Mamisashvili L, Tomlinson G, Dennis CL, Koren $\mathrm{G}$, et al. The impact of maternal depression during pregnancy on perinatal outcomes: a systematic review and meta-analysis. J Clin Psychiatry. 2013;74(4):e321-e41.

23. Bell AF, Andersson E. The birth experience and women's postnatal depression: a systematic review. Midwifery. 2016;39:112-23.

24. Chapman SLC, Wu L-T. Postpartum substance use and depressive symptoms: a review. Women Health. 2013:53(5):479-503.

25. Sawyer A, Ayers S, Smith H. Pre- and postnatal psychological wellbeing in Africa: a systematic review. J Affect Disord. 2010;123(1-3):17-29.

26. Vigod $S N$, Villegas $L$, Dennis $C L$, Ross LE. Prevalence and risk factors for postpartum depression among women with preterm and low-birth-weight infants: a systematic review. BJOG Int J Obstet Gynaecol. 2010;117(5):540-50.
27. Villegas L, McKay K, Dennis C-L, Ross LE. Postpartum depression among rural women from developed and developing countries: a systematic review. J Rural Health. 2011;27(3):278-88.

28. Wittkowski A, Gardner PL, Bunton P, Edge D. Culturally determined risk factors for postnatal depression in sub-Saharan Africa: a mixed method systematic review; 2014

29. Fekadu Dadi A, Miller ER, Mwanri L. Antenatal depression and its association with adverse birth outcomes in low and middle-income countries: a systematic review and meta-analysis. PLoS One. 2020;15(1):e0227323.

30. Galbraith RF. Graphical display of estimates having differing standard errors. Technometrics. 1988;30(3):271-81.

31. Higgins J, Thompson S, Deeks J, Altman D. Statistical heterogeneity in systematic reviews of clinical trials: a critical appraisal of guidelines and practice. J Health Serv Res Policy. 2002;7(1):51-61.

32. Egger M, Davey Smith G, Schneider M, Minder C. Bias in meta-analysis detected by a simple, graphical test. BMJ. 1997;315(7109):629-34.

33. Ioannidis JP. Interpretation of tests of heterogeneity and bias in metaanalysis. J Eval Clin Pract. 2008;14(5):951-7.

34. Duval S, Tweedie R. Trim and fill: a simple funnel-plot-based method of testing and adjusting for publication bias in meta-analysis. Biometrics. 2000; 56(2):455-63.

35. Nguyen DTN, Hughes S, Egger S, LaMontagne DS, Simms K, Castle PE, et al. Risk of childhood mortality associated with death of a mother in low-andmiddle-income countries: a systematic review and meta-analysis. BMC Public Health. 2019;19(1):1281.

36. Stare J, Maucort-Boulch D. Odds ratio, hazard ratio and relative risk. Metodoloski zvezki. 2016;13(1):59.

37. Cummings $P$. The relative merits of risk ratios and odds ratios. Arch Pediatr Adolesc Med. 2009;163(5):438-45.

38. Stata A. Stata Base reference manual release 14; 2015.

39. Abdollahi F, Etemadinezhad S, Lye MS. Postpartum mental health in relation to sociocultural practices. Taiwanese J Obstetr Gynecol. 2016;55(1):76-80.

40. Abdollahi F, Zarghami M, Azhar MZ, Sazlina SG, Lye MS. Predictors and incidence of post-partum depression: a longitudinal cohort study. J Obstet Gynaecol Res. 2014;40(12):2191-200.

41. Ahmed HM, Alalaf SK, Al-Tawil NG. Screening for postpartum depression using Kurdish version of Edinburgh postnatal depression scale; 2012.

42. Azale T, Fekadu A, Hanlon C. Treatment gap and help-seeking for postpartum depression in a rural African setting. BMC Psychiatry. 2016;16(1): 196.

43. Baghianimoghadam MH, Shodjaee Zadeh D, Aminian AH. Caesarean section, vaginal delivery and post natal depression. Iran J Public Health. 2009:38(3):118-22 Date of Publication: 2009.; 2009.

44. Bottino MN, Nadanovsky P, Moraes CL, Reichenheim ME, Lobato G. Reappraising the relationship between maternal age and postpartum depression according to the evolutionary theory: empirical evidence from a survey in primary health services. J Affect Disord. 2012;142(1-3):219-24.

45. Correa H, Castro e Couto T, Santos W, Romano-Silva M, Santos L. Postpartum depression symptoms among Amazonian and northeast Brazilian women. J Affect Disord. 2016;204:214-8.

46. de Castro F, Place JM, Billings DL, Rivera L, Frongillo EA. Risk profiles associated with postnatal depressive symptoms among women in a public sector hospital in Mexico: the role of sociodemographic and psychosocial factors. Arch Womens Ment Health. 2015;18(3):463-71.

47. Deng A-W, Xiong R-B, Jiang T-T, Luo Y-P, Chen W-Z. Prevalence and risk factors of postpartum depression in a population-based sample of women in Tangxia community, Guangzhou. Asian Pac J Trop Med. 2014;7(3):244-9.

48. Dindar I, Erdogan S. Screening of Turkish women for postpartum depression within the first postpartum year: the risk profile of a community sample. Public Health Nurs. 2007;24(2):176-83.

49. Durat $G$, Kutlu Y. The prevalence of postpartum depression and related factors in Sakarya. Yeni Symposium. 2010;48(1):63-9.

50. Ege E, Timur S, Zincir H, Geckil E, Sunar-Reeder B. Social support and symptoms of postpartum depression among new mothers in eastern Turkey. J Obstet Gynaecol Res. 2008;34(4):585-93.

51. El-Hachem C, Rohayem J, Bou Khalil R, Richa S, Kesrouani A, Gemayel R, et al. Early identification of women at risk of postpartum depression using the Edinburgh postnatal depression scale (EPDS) in a sample of Lebanese women. BMC Psychiatry. 2014;14:242.

52. Flores-Quijano ME, Cordova A, Contreras-Ramirez V, Farias-Hernandez L Cruz Tolentino M, Casanueva E. Risk for postpartum depression, 
breastfeeding practices, and mammary gland permeability. J Hum Lact. 2008;24(1):50-7.

53. Gao L-I, Chan SW-C, Mao Q. Depression, perceived stress, and social support among first-time Chinese mothers and fathers in the postpartum period. Res Nurs Health. 2009;32(1):50-8.

54. Giri RK, Khatri RB, Mishra SR, Khanal V, Sharma VD, Gartoula RP. Prevalence and factors associated with depressive symptoms among post-partum mothers in Nepal. BMC Res Notes. 2015;8:111.

55. Goker A, Yanikkerem E, Demet MM, Dikayak S, Yildirim Y, Koyuncu FM. Postpartum depression: is mode of delivery a risk factor? ISRN Obstetr Gynecol. 2012;2012:6.

56. Guo N, Bindt C, Te Bonle M, Appiah-Poku J, Hinz R, Barthel D, et al. Association of antepartum and postpartum depression in Ghanaian and Ivorian women with febrile illness in their offspring: a prospective birth cohort study. Am J Epidemiol. 2013;178(9):1394-402.

57. Hassanein IMA, Fathalla MMF, Abdel RT. The role of newborn gender in postpartum depressive symptoms among women in upper Egypt. Int J Gynecol Obstet. 2014;125(2):138-40.

58. Hasselmann MH, Werneck GL, Silva CV. Symptoms of postpartum depression and early interruption of exclusive breastfeeding in the first two months of life. Cad Saude Publica. 2008:24(Suppl 2):S341-52.

59. Ho-Yen SD, Bondevik GT, Eberhard-Gran M, Bjorvatn B. Factors associated with depressive symptoms among postnatal women in Nepal; 2007.

60. Iranpour S, Kheirabadi GR, Heidari-Beni M, Maracy MR. Association between Caffeine Consumption during Pregnancy and Postpartum Depression: A Population-Based Study. J Caffeine Res. 2017;7(1):1-6 Date of Publication: March 2017:; 2017.

61. Kadir AA, Daud MNM, Yaacob M, Hussain NHN. Relationship between obstetric risk factors and postnatal depression in Malaysian women. Int Med J. 2009:16(2):101-6

62. Khalifa DS, Glavin K, Bjertness E, Lien L. Postnatal depression among Sudanese women: Prevalence and validation of the Edinburgh Postnatal depression scale at 3 months postpartum. Int J Womens Health. 2015;7: 677-84 Date of Publication: 08 Jul 2015.; 2015.

63. Lara MA, Navarrete L, Nieto L, Barba Martín JP, Navarro JL, Lara-Tapia H. Prevalence and incidence of perinatal depression and depressive symptoms among Mexican women. J Affect Disord. 2015;175:18-24.

64. Liu S, Yan Y, Gao X, Xiang S, Sha T, Zeng G, et al. Risk factors for postpartum depression among Chinese women: path model analysis. BMC Pregnancy Childbirth. 2017;17(1):133

65. Mathisen SE, Glavin K, Lien L, Lagerlov P. Prevalence and risk factors for postpartum depressive symptoms in Argentina: a cross-sectional study. Int J Women's Health. 2013;5:787-93.

66. Melo EF Jr, Cecatti JG, Pacagnella RC, Leite DFB, Vulcani DE, Makuch MY The prevalence of perinatal depression and its associated factors in two different settings in Brazil. J Affect Disord. 2012;136(3):1204-8.

67. Mohammed ES, Mosalem FA, Mahfouz EM, Abd ElHameed MA. Predictors of postpartum depression among rural women in Minia, Egypt: an epidemiological study. Public Health. 2014;128(9):817-24.

68. Murray L, Dunne MP, Van Vo T, Anh PN, Khawaja NG, Cao TN. Postnatal depressive symptoms amongst women in Central Vietnam: a cross-sectional study investigating prevalence and associations with social, cultural and infant factors. BMC Pregnancy Childbirth. 2015:15:234.

69. Panyayong B. Postpartum depression among Thai women: a national survey; 2013.

70. Petrosyan D, Armenian HK, Arzoumanian K. Interaction of maternal age and mode of delivery in the development of postpartum depression in Yerevan, Armenia. J Affect Disord. 2011;135(1-3):77-81.

71. Ramchandani PG, Richter LM, Stein A, Norris SA. Predictors of postnatal depression in an urban south African cohort. J Affect Disord. 2009;113(3): 279-84.

72. Safadi RR, Abushaikha LA, Ahmad MM. Demographic, maternal, and infant health correlates of post-partum depression in Jordan. Nurs Health Sci. 2016:18(3):306-13.

73. Serhan N, Ege E, Ayrancı U, Kosgeroglu N. Prevalence of postpartum depression in mothers and fathers and its correlates. J Clin Nurs. 2013;22(1/ 2):279-84

74. Shamu S, Zarowsky C, Roelens K, Temmerman M, Abrahams N. Highfrequency intimate partner violence during pregnancy, postnatal depression and suicidal tendencies in Harare, Zimbabwe. Gen Hosp Psychiatry. 2016;38: 109-14.
75. Shivalli S, Gururaj N. Postnatal depression among rural women in South India: do socio-demographic, obstetric and pregnancy outcome have a role to play? PLoS One. 2015;10(4):e0122079.

76. Stellenberg EL, Abrahams JM. Prevalence of and factors influencing postnatal depression in a rural community in South Africa. Afr J Primary Health Care Fam Med. 2015;7(1):874.

77. Stewart RC, Bunn J, Vokhiwa M, Umar E, Kauye F, Fitzgerald M, et al. Common mental disorder and associated factors amongst women with young infants in rural Malawi. Soc Psychiatry Psychiatr Epidemiol. 2010;45(5): 551-9.

78. Tannous L, Gigante LP, Fuchs SC, Busnello ED. Postnatal depression in Southern Brazil: Prevalence and its demographic and socioeconomic determinants. BMC Psychiatry. 2008;8:ArtID 1.

79. Wan EY, Moyer CA, Harlow SD, Fan Z, Jie Y, Yang H. Postpartum depression and traditional postpartum care in China: role of zuoyuezi; 2009.

80. Weobong B, Ten Asbroek AH, Soremekun S, Danso S, Owusu-Agyei S, Prince $M$, et al. Determinants of postnatal depression in rural Ghana. Depress Anxiety. 2013;32(2):108-19.

81. Yagmur Y, Ulukoca N. Social support and postpartum depression in lowsocioeconomic level postpartum women in eastern Turkey. Int J Public Health. 2010:55(6):543-9.

82. Zainal NZ, Kaka AS, Ng CG, Jawan R, Singh GJ. Prevalence of postpartum depression in a hospital setting among Malaysian mothers. Asia Pac Psychiatry. 2012;4(2):144-9.

83. Gausia K, Ali M, Ryder D. Diarrhea in Bangladeshi infants and its association with postnatal depression. Bangladesh Med Res Counc Bull. 2010;36(1):32-4.

84. Gupta S, Kishore J, Mala YM, Ramji S, Aggarwal R. Postpartum depression in north Indian women: prevalence and risk factors. J Obstetr Gynaecol India. 2013;63(4):223-9.

85. Islam MJ, Baird K, Mazerolle P, Broidy L. Exploring the influence of psychosocial factors on exclusive breastfeeding in Bangladesh. Arch Womens Ment Health. 2017:20(1):173-88.

86. Machado MCM, Assis KF, Oliveira FCC, Ribeiro AQ, Araujo RMA, Cury AF, et al. Determinants of the exclusive breastfeeding abandonment: Psychosocial factors. Revista de Saude Publica. 2014;48(6):985-94 Date of Publication: 2014: 2014

87. Noriko O, Megumi M, Hanako M, Yasuko M. Birth experience and postnatal depression: Women's negative evaluation as a risk factor. Asian J Nurs. 2007: 10(4):257-64 Date of Publication: December 2007:; 2007.

88. Pocan AG, Aki OE, Parlakgumus AH, Gereklioglu C, Dolgun AB. The incidence of and risk factors for postpartum depression at an urban maternity clinic in Turkey. Int J Psychiatry Med. 2013;46(2):179-94.

89. Saeed Q, Shah N, Inam S, Shafique K. Maternal depressive symptoms and child nutritional status: a cross-sectional study in socially disadvantaged Pakistani community. J Child Health Care. 2017;21(3):331-42.

90. Upadhyay AK, Srivastava S. Effect of pregnancy intention, postnatal depressive symptoms and social support on early childhood stunting: Findings from India. BMC pregnancy and childbirth. 2016;16(1).

91. Weobong B, Ten Asbroek AHA, Soremekun S, Gram L, Amenga-Etego SD, Danso S, et al. Association between probable postnatal depression and increased infant mortality and morbidity: Findings from the DON population-based cohort study in rural Ghana. BMJ Open. 2015;5(8).

92. Mohamad Yusuff AS, Tang L, Binns CW, Lee AH. Prevalence and risk factors for postnatal depression in Sabah, Malaysia: A cohort study. Women Birth. 28(1):25-9.

93. Supriya Hegde, KS Latha, Sripathy M Bhat, P.S.V.N Sharma, Asha Kamath, Avinash K. Shetty. Postpartum Depression: Prevalence and Associated Factors among Women in India. Journal of Women's Health, Issues \& Care. 2012;1(1).

94. Roberth A et, al,. Postnatal depression. 2016.

95. Surkan PJ, Kawachi I, Ryan LM, Berkman LF, Vieira LMC, Peterson KE. Maternal depressive symptoms, parenting self-efficacy, and child growth. Am J Public Health. 2008;98(1):125-32.

96. Adewuya AO, Ola BO, Aloba OO, Mapayi BM, Okeniyi JA. Impact of postnatal depression on infants' growth in Nigeria; 2008.

97. Ashaba S, Rukundo GZ, Beinempaka F, Ntaro M, Leblanc JC. Maternal depression and malnutrition in children in southwest Uganda: A case control study. BMC Public Health. 2015;15(1).

98. Madeghe BA, Kimani VN, Vander Stoep A, Nicodimos S, Kumar M. Postpartum depression and infant feeding practices in a low income urban settlement in Nairobi-Kenya. BMC Research Notes. 2016;9(1). 
99. Rahman A, Hafeez A, Bilal R, Sikander S, Malik A, Minhas F, et al. The impact of perinatal depression on exclusive breastfeeding: a cohort study. Matern Child Nutr. 2016;12(3):452-62.

100. Wemakor A, Mensah KA. Association between maternal depression and child stunting in Northern Ghana: A cross-sectional study. BMC Public Health. 2016;16(1).

101. Bennett IM, Schott W, Krutikova S, Behrman JR. Maternal mental health, and child growth and development, in four low-income and middle-income countries. J Epidemiol Community Health. 2015;70(2):168-73.

102. Black MM, Baqui AH, Zaman K, Arifeen SE, Black RE. Maternal depressive symptoms and infant growth in rural Bangladesh. Am J Clin Nutr. 2009; 89(3):951S-7S.

103. Ndokera R, MacArthur C. The relationship between maternal depression and adverse infant health outcomes in Zambia: a cross-sectional feasibility study. Child Care Health Dev. 2011;37(1):74-81.

104. Aneja S. Integrated Management of Newborn and Childhood IIIness (IMNCI) strategy and its implementation in real life situation. Indian J Pediatr. 2019; 86(7):622-7.

105. Sacher J, Wilson AA, Houle S, Rusjan P, Hassan S, Bloomfield PM, et al. Elevated brain monoamine oxidase a binding in the early postpartum period. Arch Gen Psychiatry. 2010;67(5):468-74.

106. Mathers C, Ma Fat D, JT. B. The global burden of disease: 2004 update. Geneva: World Health Organization; 2008

107. Mathers CD, Loncar D. Projections of global mortality and burden of disease from 2002 to 2030. PLoS Med. 2006;3(11):e442.

108. Affonso DD, De AK, Horowitz JA, Mayberry LJ. An international study exploring levels of postpartum depressive symptomatology. J Psychosom Res. 2000;49(3):207-16

109. Rashid A, Mohd R. Poor social support as a risk factor for antenatal depressive symptoms among women attending public antennal clinics in Penang, Malaysia. Reprod Health. 2017;14:144.

110. Howard LM, Oram S, Galley H, Trevillion K, Feder G. Domestic violence and perinatal mental disorders: a systematic review and meta-analysis. PLoS Med. 2013;10(5):e1001452.

111. Izadirad H, Niknami S, Zareban I, Hidarnia A. Effects of social support and self-efficacy on maternal prenatal cares among the first-time pregnant women, Iranshahr, Iran. J Family Reprod Health. 2017;11(2):67-73.

112. Raes F, Smets J, Wessel I, Van Den Eede F, Nelis S, Franck E, et al. Turning the pink cloud grey: dampening of positive affect predicts postpartum depressive symptoms. J Psychosom Res. 2014;77(1):64-9.

113. Lo CS, Ho SM, Hollon SD. The effects of rumination and negative cognitive styles on depression: a mediation analysis. Behav Res Ther 2008;46(4):487-95.

114. Barnum SE, Woody ML, Gibb BE. Predicting changes in depressive symptoms from pregnancy to postpartum: the role of brooding rumination and negative inferential styles. Cogn Ther Res. 2013;37:71-7.

115. McCrory EJ, Gerin MI, Viding E. Annual research review: childhood maltreatment, latent vulnerability and the shift to preventative psychiatry the contribution of functional brain imaging. J Child Psychol Psychiatry Allied Discip. 2017;58(4):338-57.

116. Teicher $\mathrm{MH}$, Parigger A. The 'Maltreatment and abuse chronology of Exposure' (MACE) scale for the retrospective assessment of abuse and neglect during development. PLoS One. 2015;10(2):e0117423.

117. Kaaya SF, Mbwambo JK, Kilonzo GP, Van Den Borne H, Leshabari MT, Fawzi $M C$, et al. Socio-economic and partner relationship factors associated with antenatal depressive morbidity among pregnant women in Dar Es Salaam, Tanzania. Tanzan J Health Res. 2010;12(1):23-35.

118. Weobong B, Soremekun S, Ten Asbroek AH, Amenga-Etego S, Danso S, Owusu-Agyei S, et al. Prevalence and determinants of antenatal depression among pregnant women in a predominantly rural population in Ghana: the DON population-based study. J Affect Disord. 2014;165:1-7.

119. Surkan PJ, Patel SA, Rahman A. Preventing infant and child morbidity and mortality due to maternal depression. Best Pract Res. 2016;36:156-68.

120. Wachs TD, Black MM, Engle PL. Maternal depression: a global threat to Children's health, development, and behavior and to human rights. Child Dev Perspect. 2009;3.

121. Burns JM, Andrews G, Szabo M. Depression in young people: what causes it and can we prevent it? Med J Aust. 2002;177(Suppl):S93-6.

122. Reck C, Noe D, Stefenelli U, Fuchs T, Cenciotti F, Stehle E, et al. Interactive coordination of currently depressed inpatient mothers and their infants during the postpartum period. Infant Ment Health J. 2011;32(5):542-62.
123. Miranda V. Cooking, caring and volunteering: Unpaid work around the world. 2011.

124. de Jager E, Skouteris H, Broadbent J, Amir L, Mellor K. Psychosocial correlates of exclusive breastfeeding: a systematic review. Midwifery. 2013; 29(5):506-18.

125. Donaldson F. Postnatal depression and infant feeding: a review of the evidence. Br J Midwifery. 2011;19(10):619-24.

126. Bandura A. Self-efficacy: toward a unifying theory of behavioral change. Psychol Rev. 1977;84(2):191-215.

127. Dennis $C L$. Theoretical underpinnings of breastfeeding confidence: a selfefficacy framework. J Hum Lact. 1999;15(3):195-201.

\section{Publisher's Note}

Springer Nature remains neutral with regard to jurisdictional claims in published maps and institutional affiliations.
Ready to submit your research? Choose BMC and benefit from:

- fast, convenient online submission

- thorough peer review by experienced researchers in your field

- rapid publication on acceptance

- support for research data, including large and complex data types

- gold Open Access which fosters wider collaboration and increased citations

- maximum visibility for your research: over $100 \mathrm{M}$ website views per year

At BMC, research is always in progress.

Learn more biomedcentral.com/submissions 